Bull. Austral. Math. Soc.

VOL. 39 (1989) [301-317]

\title{
ELEMENTARY OBSERVATIONS ON 2-CATEGORICAL LIMITS
}

\author{
G.M. KELLY
}

\begin{abstract}
With a view to further applications, we give a self-contained account of indexed limits for 2-categories, including necessary and sufficient conditions for 2-categorical completeness. Many important 2-categories fail to be complete but do admit a wide class of limits. Accordingly, we introduce a variety of particular 2-categorical limits of practical importance, and show that certain of these suffice for the existence of indexed lax-and pseudo-limits. Other important 2-categories fail to admit even pseudo-limits, but do admit the weaker bilimits; we end by discussing these.
\end{abstract}

\section{INTRODUCTION}

The author and his colleagues are preparing a series of articles on two-dimensional universal algebra, the first two of which are to be [5] and [4]. These make heavy use of 2-categorical limit notions, of which there is no concerted account in print. An attempt to incorporate such an account in the first article proved so disruptive to its unity and readability that we decided to separate out this matter and discuss it in this present preliminary article.

In Section 2 we adapt to the special case $\mathcal{V}=$ Cat of 2-categories the general notion of indexed limit for $\mathcal{V}$-categories, discussed in detail in Chapter 3 of Kelly [9] - both to cater for the reader interested in 2 -categories but not in $\mathcal{V}$-categories, and to give an elementary description in this simple case. In [5] and some later articles our results need completeness (the existence of all indexed limits) and cocompleteness of the base 2-category; so in Section 3 we discuss the reduction of completeness to the existence of conical limits and cotensor products, applying this to show - a result needed in [5] - that a complete 2-category remains complete when we discard its non-invertible 2-cells. However, the 2-categories of algebras erected on such a base are not themselves complete, because the morphisms of interest are not the strict ones; yet they do admit a variety of important limits, and it behoves us to study these and their connexions.

Accordingly in Section 4 we name, and describe in elementary terms, various particular (indexed) limits peculiar to the 2-categorical case, and give relations between them - some of which are known but unpublished, while others are new: as, for instance, that powers, inserters, and equifiers suffice for cotensor products. In Section 5 ,

Received 10 May 1988

The author gratefully acknowledges the support of the Australian Research Grants Scheme.

Copyright Clearance Centre, Inc. Serial-fee code: $0004-9729 / 89 \$$ A2.00+0.00. 
we introduce, for the first time in print, (indexed) lax limits and pseudo-limits, observing that these can be exhibited as special indexed limits, and showing that they exist whenever products, inserters, and equifiers do so. This will allow us to prove in [5] that they exist in the 2-category of algebras for a 2-monad on a complete and cocomplete base 2-category, and in later articles that they exist in the 2-category of algebras for a suitable finite-limit theory.

We shall see in [5] that the 2-category of algebras for a good 2-monad on a good base need not admit pseudo-colimits; but that it does admit the (indexed) bicolimits of Street [15]. We therefore recall these in Section 6, and use a recent result of Street [16] to conclude that we can prove their existence in a 2-category by considering only special cases involving 2-categories and 2-functors, without embarking on the further complications of bicategories and their homomorphisms - an observation of great value in [5] and later papers.

We include only what is needed for the reading of our planned articles, and accordingly leave aside such examples of 2-categorical limits as the presentation in Street [13] of the Eilenberg-Moore object, and that in Street [16] of the descent object, as indexed limits. We shall in fact return to a deeper study of 2-categorical limits in [4] - to which we make one or two harmless forward references - using the results of [5].

Our references are rather to convenient compedia than to original sources. In some mitigation of this, we observe here that the notion of indexed limit for enriched categories was introduced independently by Auderset [1], Borceux and Kelly [6], and (in the special case of 2-categories) by Street [13]; and that various particular 2-categorical limits, including many of those below, were introduced by Gray in [7] (with a different nomenclature), by Street in [14] and [16], and by my student Bird in his thesis [3].

\section{INDEXED LIMITS AND COLIMITS FOE 2-CATEGORIES}

As a general reference to elementary 2-categorical notions, and for the meanings of any terms not explained below, see Kelly and Street [12]. We write Cat for the 2category of small categories; it is of course a cartesian closed category. Most 2-categories $\mathcal{K}$ met with in practice are locally small, in the sense that each $\mathcal{K}(A, B)$ is a small category; and are thus $\mathcal{V}$-categories for $\mathcal{V}=\mathbf{C a t}$. Nothing essential changes if they are not locally small - they are then CAT-categories for the CAT of a higher universe. Discarding the 2-cells of a 2-category $\mathcal{K}$ gives its underlying ordinary category $\mathcal{K}_{0}$.

As is usual with $\mathcal{V}$-categories, we abbreviate as follows wherever convenient. If $\mathcal{K}$ and $\mathcal{L}$ are 2-categories, by a functor $G: \mathcal{K} \rightarrow \mathcal{L}$ we mean a 2-functor (that is, a Cat-functor; for a description in elementary terms, see [12]). If we wanted to speak of a mere functor $\mathcal{K}_{0} \rightarrow \mathcal{L}_{0}$ between their underlying categories, we should say just this. Similarly, by a natural transformation $\alpha: G \rightarrow H: \mathcal{K} \rightarrow \mathcal{L}$, we mean a 2-natural one; see [12] again. If we speak of a 2-functor $G: \mathcal{K} \rightarrow \mathcal{L}$, we imply that $\mathcal{K}$ and $\mathcal{L}$ are 
2-categories. A 2-functor $F: \mathcal{C} \rightarrow$ Cat is representable when, for some $D \in \mathcal{C}$, there is a 2-natural isomorphism $\mathcal{C}(D, C) \cong F C$ of categories. The 2-functor $G: \mathcal{K} \rightarrow \mathcal{L}$ has a left adjoint when there is a 2-functor $S: \mathcal{L} \rightarrow \mathcal{K}$ and a 2-natural isomorphism $\mathcal{K}(S B, A) \cong \mathcal{L}(B, G A)$ of categories; this is so precisely when $\mathcal{L}(B, G-): \mathcal{K} \rightarrow$ Cat is representable for each $B$. The functor 2-category $[\mathcal{K}, \mathcal{L}]$ is the 2-category of 2-functors, 2-natural transformations, and modifications; for these last, see [12]. A category may be seen as a 2-category whose only 2-cells are identities (and a set as a category whose only arrows are identities).

It is well-known that, to have a sufficiently wide notion of limit for $\mathcal{V}$-categories, and an appropriate concept of complete $\mathcal{V}$-category, one needs the indexed limits of $[\boldsymbol{\theta}$, Chapter 3] (which some authors now prefer to call weighted limits). We here recall the general notion in the case $\mathcal{V}=$ Cat. An indexing type is a 2-functor $F: \mathcal{P} \rightarrow$ Cat where (for us) $\mathcal{P}$ is small. A 2-functor $G: \mathcal{P} \rightarrow \mathcal{K}$ gives rise to a 2 -functor $\mathcal{K}^{\text {op }} \rightarrow$ Cat sending $A$ to the right side of (2.1) below; if this latter 2 -functor admits a representation

$$
\mathcal{K}(A,\{F, G\}) \cong[\mathcal{P}, \mathbf{C a t}](F, \mathcal{K}(A, G-))
$$

with unit say

$$
\xi: F \rightarrow \mathcal{K}(\{F, G\}, G-)
$$

we call the object $\{F, G\}$ of $\mathcal{K}$, or more properly the pair $(\{F, G\}, \xi)$, the $F$-indexed limit of $G$. (The reader to whom this definition is unfamiliar should find clarification of its scope and its meaning in the particular examples of the following sections.)

Observe that the functor from the left to the right side of (2.1) induced by the unit $\xi$ is required to be an isomorphism of categories, and not just an isomorphism

$$
\mathcal{K}_{0}(A,\{F, G\}) \cong[\mathcal{P}, \text { Cat }]_{0}(F, \mathcal{K}(A, G-))
$$

of sets. The isomorphism (2.3), asserting that every natural $\rho: F \rightarrow \mathcal{K}(A, G-)$ is $\mathcal{K}(h, G-) \xi$ for a unique arrow $h: A \rightarrow\{F, G\}$ in $\mathcal{K}$, expresses what we may call the one-dimensional aspect of the universal property of $\{F, G\}$; but (2.1) expresses a two-dimensional aspect as well: every modification $\theta: \rho \rightarrow \rho^{\prime}$ is $\mathcal{K}(\alpha, G-) \xi$ for a unique 2-cell $\alpha: h \rightarrow h^{\prime}$. (Note that $\alpha$ is invertible if and only if $\theta$ is so.) Examples in Sections 3.7 and 3.8 of [9] show that the one-dimensional aspect (2.3) does not in general imply the two-dimensional one, and hence does not suffice to exhibit a candidate $(\{F, G\}, \xi)$ as the limit. It does, however, suffice to determine $(\{F, G\}, \xi)$ uniquely to within isomorphism, since the Set-valued functor on the right of $(2.3)$ is represented by $\{F, G\}$ with unit $\xi$. 
The 2-category $\mathcal{K}$ is complete when it admits all limits $\{F, G\}$ (with $\mathcal{P}$ small). If we write $\widehat{G}: \mathcal{K}^{o p} \rightarrow[\mathcal{P}$, Cat $]$ for the 2 -functor given by

$$
\widehat{G} A=\mathcal{K}(A, G-),
$$

to say that $\mathcal{K}$ is complete is to say that, for all $\mathcal{P}$ and all $G: \mathcal{P} \rightarrow \mathcal{K}$, the functor $\widehat{G}$ has a left adjoint $\{-, G\}$; then the $\xi$ of $(2.2)$ is the $F$-component of the unit of the adjunction.

The 2-category Cat is complete, an easy calculation showing that (2.1) is satisfied by

$$
\{F, G\}=[\mathcal{P}, \text { Cat }](F, G) \text { when } \mathcal{K}=\text { Cat }
$$

with the evident unit. Accordingly we can re-write (2.1) as

$$
\mathcal{K}(A,\{F, G\}) \cong\{F, \mathcal{K}(A, G-)\},
$$

exhibiting the definition of limit (like that of classical limits) as a representable one.

Colimits in $\mathcal{K}$ are just limits in $\mathcal{K}^{o p}$. The notation for the colimit of $G: \mathcal{P} \rightarrow \mathcal{K}$ indexed by $F: \mathcal{P}^{o p} \rightarrow$ Cat (note the variance) is $F * G$, and (2.1) becomes

$$
\mathcal{K}(F * G, A) \cong\left[\mathcal{P}^{o p}, \mathbf{C a t}\right](F, \mathcal{K}(G-, A)) .
$$

The analogue of the $\widehat{G}$ of $(2.4)$ is now $\tilde{G}: \mathcal{K} \rightarrow\left[\mathcal{P}^{\text {op }}\right.$, Cat $]$ given by

$$
\widetilde{G} A=\mathcal{K}(G-, A),
$$

and $\mathcal{K}$ is cocomplete precisely when $\widetilde{G}$ has a left adjoint $-* G$ for all $G: \mathcal{P} \rightarrow \mathcal{K}$.

When $\mathcal{K}=$ Cat, so that in (2.7) we have $F: \mathcal{P}^{o p} \rightarrow$ Cat and $G: \mathcal{P} \rightarrow$ Cat, both $F * G$ and $G * F$ make sense; they are canonically isomorphic by (2.7) and the 2-natural isonorphism $[F P,[G Q, A]] \cong[G Q,[F P, A]]$, where $[X, Y]=\operatorname{Cat}(X, Y)$ is the internal hom in Cat.

Finally, taking $F$ to be representable, the Yoneda Lemma $[\mathcal{P}, \operatorname{Cat}](\mathcal{P}(P,-), H) \cong$ $H P$ (see Section 2.4 of $[9]$ ), along with (2.1) and (2.7), gives the Yoneda isomorphisms

$$
\{\mathcal{P}(P,-), G\} \cong G P, \mathcal{P}(-, P) * G \cong G P .
$$

3. CONICAL LIMITS, COTENSOR PRODUCTS, AND COMPLETENESS

It was shown in Chapter 3 of [9] that a $\mathcal{V}$-category is complete if and only if it admits conical limits and cotensor products; or equivalently products, equalizers, and cotensor products - all of these being particular indexed limits. We give here a brief 
independent discussion of this in the 2-categorical case, and an application (needed in [5]) to groupoid-enriched categories.

When $F$ is the 2-functor $\Delta 1: \mathcal{P} \rightarrow$ Cat constant at the unit category 1 , and $G: \mathcal{P} \rightarrow \mathcal{K}$ is any 2 -functor, we write $\lim G$ for $\{\Delta 1, G\}$, and call such limits conical. The point is that to give an object of the right side of (2.1) with $F=\Delta 1$ is equally to give a (projective) cone over $G$ with vertex $A$, and to give a morphism is to give a modification of such cones; while the unit $\xi$ in (2.2) corresponds to a particular cone $\bar{\xi}$ over $G$ with vertex $\lim G$. The one-dimensional aspect of the universal property asserts that any cone over $G$ with vertex $A$ is $\bar{\xi} h$ for a unique $h: A \rightarrow \lim G$, while the two-dimensional aspect asserts that any modification $\bar{\xi} h \rightarrow \bar{\xi} k$ is $\bar{\xi} \alpha$ for a unique 2-cell $\alpha: h \rightarrow k$. When $\mathcal{P}$ is a category rather than a general 2-category, the onedimensional aspect is clearly the assertion that $(\lim G, \bar{\xi})$ is the classical limit in the category $\mathcal{K}_{0}$ of the functor $G: \mathcal{P} \rightarrow \mathcal{K}_{0}$; but when we call $\lim G$ the limit of $G$ in $\mathcal{K}$ (and not merely in $\mathcal{K}_{0}$ ), we affirm the two-dimensional aspect as well.

Among such conical limits (with $\mathcal{P}$ a category) are the various familiar ones: products, equalizers, pullbacks, and so on. An example in Section 3.8 of [9] shows that even a binary product in $\mathcal{K}_{0}$ need not be a product in $\mathcal{K}$.

We write, of course, $\operatorname{colim} G$ for the conical limit in $\mathcal{K}^{o p}$ of $G^{o p}: \mathcal{P}^{o p} \rightarrow \mathcal{K}^{o p}$, namely $\triangle 1 * G$.

When we take for $\mathcal{P}$ in (2.1) the unit category 1 , we may identify $F$ and $G$ with objects of Cat and of $\mathcal{K}$; then $\{F, G\}$ is called the cotensor product of $F$ and $G$ (and $F * G$ is called the tensor product). The universal properties (2.1) and (2.7) become

$$
\mathcal{K}(A,\{F, G\}) \cong[F, \mathcal{K}(A, G)], \quad \mathcal{K}(F * G, A) \cong[F, \mathcal{K}(G, A)]
$$

here $[X, Y]$ is again the internal hom in Cat. When $\mathcal{K}=$ Cat, we have $\{F, G\}=$ $[F, G]$ by (2.5); and we clearly have $F * G=F \times G$.

It is useful, both for this article and for [5], to have a description in elementary terms of the universal property of the cotensor product $\{F, G\}$. To give the unit $\xi: F \rightarrow \mathcal{K}(\{F, G\}, G)$ is to give maps $\xi_{x}:\{F, G\} \rightarrow G$ in $\mathcal{K}$ for each object $x$ of the category $F$, and to give 2-cells $\xi_{\phi}: \xi_{x} \rightarrow \xi_{y}$ in $\mathcal{K}$ for each $\phi: x \rightarrow y$ in $F$, these data making $\xi$ into a functor. The one-dimensional aspect of the universal property asserts that, whenever we have maps $f_{x}: A \rightarrow G$ and 2-cells $f_{\phi}: f_{x} \rightarrow f_{y}$ making $f$ into a functor $F \rightarrow \mathcal{K}(A, G)$, there is a unique map $h: A \rightarrow\{F, G\}$ such that $\xi_{x} h=f_{x}$ and $\xi_{\phi} h=f_{\phi}$. The two-dimensional aspect asserts that, given $h, k: A \rightarrow\{F, G\}$ and 2-cells $\beta_{x}: \xi_{x} h \rightarrow \xi_{x} k$ for each $x \in F$, satisfying $\left(\xi_{\phi} k\right) \beta_{x}=\beta_{y}\left(\xi_{\phi} h\right)$ for each $\phi: x \rightarrow y$ in $F$, there is a unique 2-cell $\alpha: h \rightarrow k$ with $\xi_{x} \alpha=\beta_{x}$ for all $x$.

The reader should note, for future purposes, the simplified version of this description in the special cases where $F=2$, by which we denote the arrow-category $0 \rightarrow 1$, 
and where $F=\mathbf{I}$, the category with objects 0 and 1 and with inverse isomorphisms $0 \rightarrow 1$ and $1 \rightarrow 0$. Observe that, when we write $0: 1 \rightarrow 2$ and $1: 1 \rightarrow 2$ below, we mean the functors which are the names of $0 \in 2$ and $1 \in 2$; the context makes it clear that $1: 1 \rightarrow 2$ cannot denote an identity functor.

When $\mathcal{K}$ admits tensor products of the form $2 * A$, the two-dimensional universal property of any limit does follow from the one-dimensional one. For the unit $\xi$ of the representation (2.3) induces a functor $p$ from the left to right side of $(2.1)$; $\mathbf{C a t}_{0}(2, p)$ is invertible since it is in effect the isomorphism (2.3) with $2 * A$ in place of $A$; and Cat $_{0}(2,-):$ Cat $_{0} \rightarrow$ Set reflects isomorphisms. (See Theorem 4.85 of [8].)

The 2-category $\mathcal{K}$ is complete if and only if it admits products, cotensor products, and equalizers; for the general $\{F, G\}$ of $(2.1)$ can be constructed as the equalizer of $v$ and $w$ in

$$
\{F, G\} \underset{u}{\longrightarrow} \Pi_{P \in \mathcal{P}}\{F P, G P\} \underset{w}{\stackrel{v}{\rightrightarrows}} \Pi_{P, Q \in \mathcal{P}}\{\mathcal{P}(P, Q) \times F P, G Q\}
$$

where $v, w$ are the evident maps arising from $\mathcal{P}(P, Q) \rightarrow \mathcal{K}(G P, G Q)$ and $\mathcal{P}(P, Q) \rightarrow$ $[F P, F Q]$. To verify such a statement, asserting that a limit may be constructed by combining other limits, it suffices, because of the representable nature of limits expressed by (2.6), to carry out the verification when $\mathcal{K}=$ Cat - which, given (2.5), is easy.

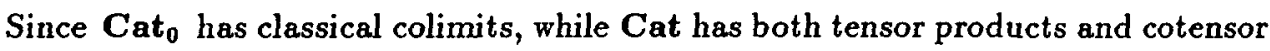
products, it follows from the last two paragraphs that Cat (which we have seen to be complete) is also cocomplete.

An example in Section 3.8 of [9] shows that $\mathcal{K}$ may admit all conical limits even those where $\mathcal{P}$ is not just a category but a general 2-category - but fail to be complete. However a $\mathcal{K}$ with products and equalizers is complete if it admits those cotensor products of the form $\{2, B\}$, since the general cotensor product $\{X, B\}$ can be constructed from these using products and equalizers. This is because, 2 being a strong generator of $\mathbf{C a t}_{0}$, every small category $X$ is in the closure of 2 under coproducts and coequalizers in $\mathbf{C a t}_{0}$ and hence, by the last paragraph, in Cat; while $\{-, B\}$ sends colimits into limits.

We call $\mathcal{K}$ finitely complete if it admits finite products, equalizers, and the cotensor products $\{2, B\}$; by Section 4 of Kelly $[10]$ it then admits the limit $\{F, G\}$ whenever ob $\mathcal{P}$ is finite and each category $\mathcal{P}(P, Q)$ and each category $F P$ is finitely presentable.

A number of operations on 2-categories preserve completeness. It is easy to see that the functor-2-category $[\mathcal{P}, \mathcal{K}]$ is complete or cocomplete if $\mathcal{K}$ is so, limits and colimits in it being formed pointwise; a special case is the power $\mathcal{K}^{X}$ for a set $X$. Again, the usual arguments show a full reflective sub-2-category of $\mathcal{K}$ is complete or cocomplete if $\mathcal{K}$ is so; see Section 3.5 of [9]. 
The category Gpd of small groupoids is again a cartesian closed category; a Gpdcategory can be seen as a (locally-small) 2-category in which every 2-cell is invertible. Since every 2-functor between Gpd-categories is a Gpd-functor, and similarly for natural transformations and modifications, we can regard Gpd-CAT as a full sub-3-category of Cat-CAT. The inclusion has both adjoints; the right adjoint sends the 2-category $\mathcal{K}$ to the 2-category $\mathcal{K}_{g}$ obtained from $\mathcal{K}$ by leaving the objects and arrows untouched but discarding all the non-invertible 2 -cells. We need the following result in [5]:

Proposition 3.1. If the 2-category $\mathcal{K}$ is complete or cocomplete, so is the 2category $\mathcal{K}_{g}$.

Proof: It suffices to consider completeness, since $\left(\mathcal{K}^{o p}\right)_{g}=\left(\mathcal{K}_{g}\right)^{o p}$. The conical limits in $\mathcal{K}_{g}$ are just those in $\mathcal{K}$ : the one-dimensional universal property is the same assertion in $\mathcal{K}_{g}$ as in $\mathcal{K}$, while the two-dimensional one in $\mathcal{K}_{g}$ is just the restriction of that in $\mathcal{K}$ to the invertible 2-cells. The cotensor product $\{2, B\}$ in $\mathcal{K}_{g}$ is not that in $\mathcal{K}$, the unit $\xi$ of which involves two maps $\xi_{0}, \xi_{1}:\{2, B\} \rightarrow B$ and a mere (not invertible) 2-cell $\lambda: \xi_{0} \rightarrow \xi_{1}$; rather, as is very easily seen, arguing about the universal properties as above, the $\{2, B\}$ of $\mathcal{K}_{g}$ is the cotensor product $\{I, B\}$ in $\mathcal{K}$.

\section{SOME NOTABLE FINITE LIMITS}

We now describe and name some important finite limits which, unlike conical limits and cotensor products, are peculiar to the case $\mathcal{V}=$ Cat of 2-categories. Typically, the components of the unit $\xi$ in (2.2) are determined, given $G$, by some subset of them; we write only the independent ones (such as $p$ and $\lambda$ in our first example).

When $\mathcal{P}$ and its images under $F$ and $G$ are

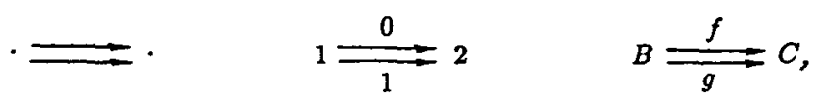

the limit is the universal diagram of the form

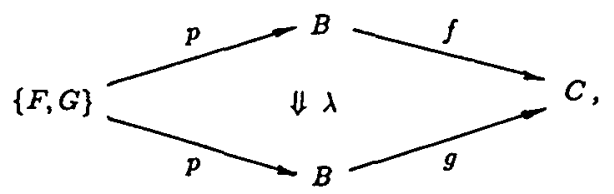

called the inserter of $f$ and $g$. The one-dimensional aspect of its universal property asserts that, given any $q: A \rightarrow B$ and any 2-cell $\mu: f q \rightarrow g q$, there is a unique $h: A \rightarrow\{F, G\}$ satisfying $p h=q$ and $\lambda h=\mu$. The two-dimensional aspect asserts 
that, given $h, k: A \rightarrow\{F, G\}$ and a 2-cell $\beta: p h \rightarrow p k$ satisfying $(\lambda k)(f \beta)=(g \beta)(\lambda h)$, there is a unique 2-cell $\alpha: h \rightarrow k$ with $p \alpha=\beta$. If in the description above of $F$ we replace 2 by $I$, we get the iso-inserter; now $\lambda$ in (4.1) is invertible, and the onedimensional universal property holds for invertible $\mu$ (but the two-dimensional one still for any $\beta$, invertible or not). Of course, replacing 2 not by $I$ but by 1 give the equalizer of $f$ and $g$.

When $\mathcal{P}$ and its images under $F$ and $G$ are

$$
\longrightarrow \cdot \longleftarrow \cdot 1 \underset{0}{\longrightarrow} 2 \underset{1}{\leftarrow} \quad B \underset{f}{\longrightarrow} D \stackrel{\leftarrow}{\longrightarrow} C
$$

the limit

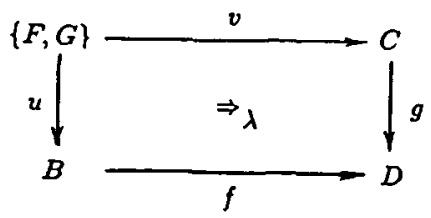

is called the comma-object of $f$ and $g$; here common notations for $\{F, G\}$ are $f / g$ and $f \downarrow g$. If we replace 2 here by $\mathbf{I}$, we get instead the iso-comma-object: the universal diagram of the form (4.2) with $\lambda$ invertible. We leave it to the reader to spell out the universal property.

When $\mathcal{P}$ and its images under $F$ and $G$ are

$$
\longrightarrow \cdot 1 \underset{0}{\longrightarrow} 2 \quad B \underset{f}{\longrightarrow} D
$$

the limit is the universal diagram of the form

(4.3)

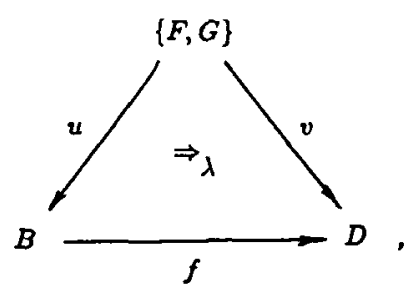

called the lax limit of the arrow $f$. Changing $F$ to $1: 1 \rightarrow 2$, and so reversing the sense of $\lambda$ in (4.3), gives the op-lax limit of $f$; while changing $F$ to $0: 1 \rightarrow I$, so that the $\lambda$ of (4.3) is invertible, gives the pseudo-limit of $f$. 
The reader will note that the lax limit (4.3) is the special case of the comma-object (4.2) in which $g$ is $1: D \rightarrow D$; and that the cotensor product $\{2, D\}$ is the still more special case in which both $f$ and $g$ are $1_{D}$. The general principle about one limit's appearing as a special case of another is the following, which is Proposition 4.57 of [9]; here $\operatorname{Lan}_{N} F$ is the left Kan extension of $F$ along $N$. Given $F: \mathcal{P} \rightarrow$ Cat, $N: \mathcal{P} \rightarrow \mathcal{Q}$, and $H: \mathcal{Q} \rightarrow \mathcal{K}$, we have

$$
\left\{\operatorname{Lan}_{N} F, H\right\} \cong\{F, H N\}
$$

either existing if the other does.

The following simple observation is well known:

Proposition 4.1. If $\mathcal{K}$ admits binary products and inserters [iso-inserters], it admits comma-objects [iso-comma-objects] and their special cases: the lax limit and the op-lax limit [the pseudo-limit] of an arrow, and the cotensor product $\{2, B\}$ [the cotensor product $\{\mathbf{I}, B\}]$.

Proof: Taking the product $B \times C$ with its projections $p$ and $q$, we find the comma-object (4.2) as the inserter of $f p$ and $g q$; as usual it suffices to verify this when $\mathcal{K}=$ Cat , which it is easy to do.

When $\mathcal{P}$ and its images under $F$ and $G$ are
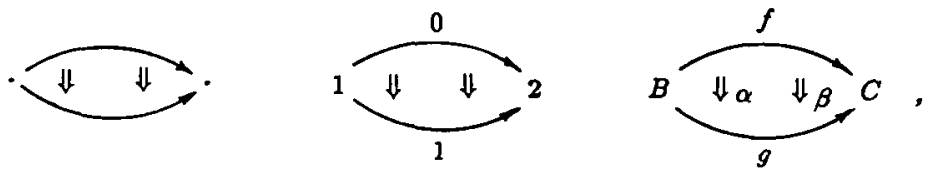

the limit

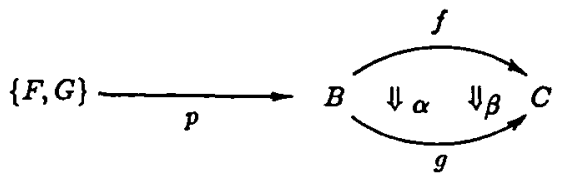

is the equifier of $\alpha$ and $\beta$. Here $p$ is universal with the property that $\alpha p=\beta p$; given any $q: A \rightarrow B$ with $\alpha q=\beta q$, there is a unique $h: A \rightarrow\{F, G\}$ satisfying $p h=q$. The two-dimensional aspect is the assertion that, given $h, k: A \rightarrow\{F, G\}$ and any 2-cell $\mu: p h \rightarrow p k$, there is a unique 2-cell $\lambda: h \rightarrow k$ satisfying $p \lambda=\mu$. 
When $\mathcal{P}$ and its images under $F$ and $G$ are
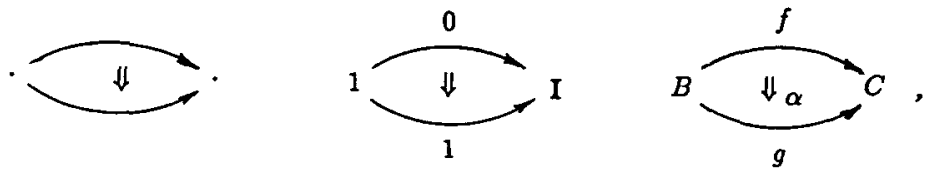

the limit

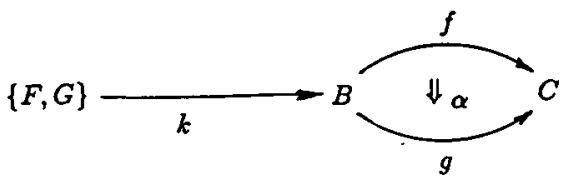

is the inverter of $\alpha$; here $k$ is universal with the property that $\alpha k$ is invertible. If we replace $I$ here by 1 , we get the identifier of $\alpha:$ a map $k:\{F, G\} \rightarrow B$ as in (4.6), but now universal with the properties $f k=g k$ and $\alpha k=$ identity.

The identifier, like the equalizer, stands apart from the other limits we have introduced in this section: the latter do exist, but equalizers and identifiers do not, in the 2-category Lex of finitely-complete categories, left-exact functors, and natural transformations - see [5]. (Note that a 2-category which admits inserters and identifiers must admit equalizers: if (4.1) is the inserter of $f$ and $g$ and $k$ is the identifier of $\lambda$, then $p k$ is the equalizer of $f$ and $g$.) The condition $f k=g k$ in the definition of the identifier, and the corresponding condition for the equalizer, demanding actual equality of two maps, make these limits of a "tight" kind, unapt to exist in such 2-categories as Lex.

This "tightness" is lacking in the endo-identifier, which is the special case of the identifier (4.6) in which $g=f$. This can of course be expressed as a limit in its own right: the indexing type is $\Delta 1: \mathcal{P} \rightarrow$ Cat, where the underlying category of $\mathcal{P}$ is $\rightarrow$. and the 2-cells are the powers of a free endomorphism of the arrow. Similarly for the auto-identifier - the identifier of an invertible 2-cell $\alpha: f \rightarrow f: B \rightarrow C$.

Most of the following is in Bird's thesis [3]:

Proposimion 4.2. (a) If equifiers exist in a 2-category, so do endo-identifiers; (b) if inserters and endo-identifiers exist, so do inverters; (c) if inserters and inverters exist, so do iso-inserters. Hence a 2-category with inserters and equifiers admits endo-identifiers, inverters, and iso-inserters; if it also admits binary products, it admits comma-objects and iso-comma-objects, the lax limit, the op-lax limit, and the pseudo-limit of an arrow, and the cotensor products $\{2, B\}$ and $\{I, B\}$.

Proof: For (a), the endo-identifier of $\alpha: f \rightarrow f: B \rightarrow C$ is the equifier of $\alpha$ and the identity. For (b), consider $\alpha: f \rightarrow g: B \rightarrow C$; form the inserter $u: D \rightarrow B$, 
$\beta: g u \rightarrow f u$, of $g$ and $f$; then the endo-identifier $v$ of $\beta(\alpha u): f u \rightarrow f u$; and finally the endo-identifier $w$ of $(\alpha u v)(\beta v): g u v \rightarrow g u v$; now $u v w$ is the inverter of $\alpha$. For (c), consider $f, g: B \rightarrow C$; form the inserter $u: D \rightarrow B, \alpha: f u \rightarrow g u$ of $f$ and $g$; and then the inverter $v$ of $\alpha$; now $u v$ is the iso-inserter of $f$ and $g$. The last assertion follows from Proposition 4.1.

As Street points out in [16], there is a partial converse to the (a) of Proposition 4.2: when $\alpha$ is invertible, we can form the equifier of $\alpha$ and $\beta$ in (4.5) as the endo-identifier of $\alpha^{-1} \beta: f \rightarrow f$. Accordingly, if we introduce the name iso-equifier for an equifier (4.5) where both $\alpha$ and $\beta$ are invertible, we have:

Proposition 4.3. (Street) The existence of auto-identifiers is equivalent to that of iso-equifiers.

The following observation seems to be new.

Proposition 4.4. If a 2-category $\mathcal{K}$ admits products (or at least powers), inserters, and equifiers, it admits cotensor products.

Proof: Given a small category $X$, write $U$ for its set of objects, $V$ for its set of morphisms, and $W$ for the set of composable pairs $(\psi, \varphi)$ of morphisms. We have the domain and codomain maps $v, w: V \rightarrow U$, the "identities" map $i: U \rightarrow V$, and the maps $r, s, t: W \rightarrow V$ given by $r(\psi, \varphi)=\varphi, s(\psi, \varphi)=\psi \varphi, t(\psi, \varphi)=\psi$. These maps satisfy

$$
v i=w i=1, \quad v r=v s, \quad w r=v t, \quad w s=w t ;
$$

what we have, of course, is a truncated simplicial set. Given $B \in \mathcal{K}$, consider the maps $B^{v}, B^{w}: B^{U} \rightarrow B^{V}$, and let $p: C \rightarrow B^{U}$, with $\lambda: B^{v} p \rightarrow B^{w} p$, be their inserter. Let $h: D \rightarrow C$ be the equifier of the 2-cells

$$
B^{r} B^{v} p \underset{B^{r \lambda}}{\longrightarrow} B^{r} B^{w} p=B^{t} B^{v} p \underset{B^{t} \lambda}{\longrightarrow} B^{t} B^{w} p
$$

and

$$
B^{r} B^{v} p=B^{v} B^{v} p \underset{B^{v} \lambda}{\longrightarrow} B^{s} B^{w} p=B^{t} B^{w} p
$$

and let $k: E \rightarrow D$ be the equifier of the 2 -cell

$$
p h=B^{i} B^{v} p h \underset{B^{i} \lambda h}{\longrightarrow} B^{i} B^{w} p h=p h
$$

and the identity. Then $E$ is the cotensor product $\{X, B\}$, the unit $\xi: X \rightarrow \mathcal{K}(E, B)$ sending $x \in X$ to the $x$-component of $p h k$ and sending $\varphi: x \rightarrow y$ to the $\varphi$-component of $\lambda h k$. 


\section{LAX LIMITS AND PSEUDO-LIMITS}

Given 2-functors $G, H: \mathcal{P} \rightarrow \mathcal{L}$, a lax natural transformation $\rho: G \rightarrow H$ assigns to each object $P$ of $\mathcal{P}$ an arrow $\rho_{P}: G P \rightarrow H P$ and to each arrow $f: P \rightarrow Q$ in $\mathcal{P}$ a 2-cell $\rho_{f}$ as in

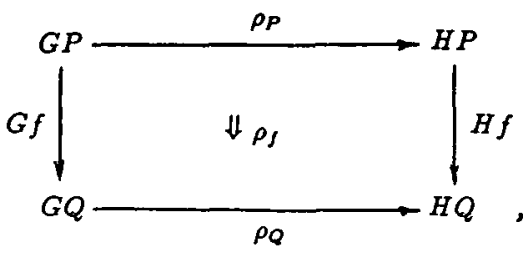

these data being subject to three axioms: one expresses the compatibility of $\rho_{f}$ and $\rho_{g}$ with $G \alpha$ and $H \alpha$ for a 2-cell $\alpha: f \rightarrow g$, the second requires $\rho_{f}$ to be the identity when $f=1_{P}$, and the third requires $\rho_{g f}$ to be the pasting composite of $\rho_{g}$ and $\rho_{f}$; for the details, see Section 2 of Kelly [8] or Section 4 of Street [14]. The lax natural $\rho$ is pseudonatural if each $\rho_{f}$ is invertible; and it is just a 2-natural transformation if each $\rho_{f}$ is the identity. The 2-functors $G: \mathcal{P} \rightarrow \mathcal{L}$, the lax natural $\rho: G \rightarrow H$, and the modifications $\theta: \rho \rightarrow \sigma: G \rightarrow H$ (see either of the references above) form a 2-category $\operatorname{Lax}[\mathcal{P}, \mathcal{L}]$. Restricting to pseudo-natural transformations gives a sub-2-category $\operatorname{Psd}[\mathcal{P}, \mathcal{L}]$, and we have locally-fully-faithful inclusions

$$
[\mathcal{P}, \mathcal{L}] \rightarrow \operatorname{Psd}[\mathcal{P}, \mathcal{L}] \rightarrow \operatorname{Lax}[\mathcal{P}, \mathcal{L}]
$$

For 2-functors $F: \mathcal{P} \rightarrow$ Cat and $G: \mathcal{P} \rightarrow \mathcal{K}$ with $\mathcal{P}$ small, the lax limit $\{F, G\}_{\ell}$ and the pseudo-limit $\{F, G\}_{p}$ of $G$ indexed by $F$ are the representing objects, if they exist, in

$$
\begin{aligned}
& \mathcal{K}\left(A,\{F, G\}_{\ell}\right) \cong \operatorname{Lax}[\mathcal{P}, \text { Cat }](F, \mathcal{K}(A, G-)) \\
& \mathcal{K}\left(A,\{F, G\}_{p}\right) \cong \operatorname{Psd}[\mathcal{P}, \text { Cat }](F, \mathcal{K}(A, G-))
\end{aligned}
$$

note that we are still asking for the representability of 2 -functors $\mathcal{K}^{o p} \rightarrow$ Cat, so that (5.3) and (5.4) are isomorphisms of categories, and not merely equivaiences. The unit of the representation (5.3) has the same general form as (2.2), namely $F \rightarrow \mathcal{K}\left(\{F, G\}_{\ell}, G-\right)$; but now of course this is only a lax natural transformation: similarly in the pseudo case. When $\mathcal{K}=$ Cat we have the analogues

$$
\{F, G\}_{\mathcal{C}}=\operatorname{Lax}[\mathcal{P}, \operatorname{Cat}](F, G), \quad\{F, G\}_{P}=\operatorname{Psd}[\mathcal{P}, \operatorname{Cat}](F, G)
$$

of (2.5); so that once again the definitions are "representable". 
Lax- and pseudo-colimits in $\mathcal{K}$ are the corresponding limits in $\mathcal{K}^{o p}$; for $F: \mathcal{P}^{o p} \rightarrow$ Cat and $G: \mathcal{P} \rightarrow \mathcal{K}$, the analogues of (2.7) are

$$
\begin{aligned}
& \mathcal{K}\left(F *_{\ell} G, A\right) \cong \operatorname{Lax}\left[\mathcal{P}^{o p}, \mathbf{C a t}\right](F, \mathcal{K}(G-, A)) \\
& \mathcal{K}\left(F *_{p} G, A\right) \cong \operatorname{Psd}\left[\mathcal{P}^{o p}, \operatorname{Cat}\right](F, \mathcal{K}(G-, A)) .
\end{aligned}
$$

Taking $F=\Delta 1: \mathcal{P} \rightarrow$ Cat gives the conical lax- and pseudo-limits, lax $\lim G=\{\triangle 1, G\}_{\ell}$ and psd $\lim G=\{\triangle 1, G\}_{p} ;$ the lax-and pseudo-limits (4.3) of an arrow are the special case $\mathcal{P}=2$. We exhibited these last two in Section 4 as ordinary indexed limits; and indeed Street showed in [14] that all conical lax- and pseudo-limits reduce to ordinary indexed linits. In fact the same is true of the general lax- and pseudo-limits of (5.3) and (5.4) which - except for some comments on pseudo-limits in Street [15] - have not hitherto been discussed in print. It will be shown in [5] that, when $\mathcal{L}$ is cocomplete, the inclusion 2 -functors $[\mathcal{P}, \mathcal{L}] \rightarrow \operatorname{Lax}[\mathcal{P}, \mathcal{L}]$ and $[\mathcal{P}, \mathcal{L}] \rightarrow \operatorname{Psd}[\mathcal{P}, \mathcal{L}]$ of (5.2) have left adjoints ()$^{\dagger}$ and ()$^{\prime}$, and these adjoints will be studied further in [4]. Taking $\mathcal{L}=$ Cat here, comparison of (5.3) and (5.4) with (2.1) gives

$$
\{F, G\}_{\ell}=\left\{F^{\dagger}, G\right\}, \quad\{F, G\}_{p}=\left\{F^{\prime}, G\right\}
$$

Accordingly pseudo-limits may be seen as those limits whose indexing type $F$ is of the form $E^{\prime}$ for some $E: \mathcal{P} \rightarrow$ Cat, and similarly for lax limits. It will be shown in [4] that inserters and equifiers are neither pseudo-limits nor lax limits; that the lax limit of an arrow is not a pseudo-limit; and that the pseudo-linit of an arrow is not a lax limit.

When $\mathcal{P}$ is a discrete category, $\operatorname{Psd}[\mathcal{P}, \mathbf{C a t}]$ and $\operatorname{Lax}[\mathcal{P}$, Cat $]$ both coincide with $[\mathcal{P}, \mathbf{C a t}]$; so pseudo-products and lax products are just products, while pseudocotensor-products and lax cotensor products are just cotensor products. The pseudo case of the following proposition is in Street [16], which corrects an error in (1.25) of his [15]; the lax case is a simple modification of his argument.

Proposition 5.1. (a) If a 2-category $\mathcal{K}$ admits products, cotensor products, inserters, and equifiers, it admits all lax limits. (b) If $\mathcal{K}$ admits products, cotensor products, iso-inserters, and iso-equifiers, it admits all pseudo-limits.

Proof: (a) Let $v$ and $w$ be the maps so denoted in (3.2). There is a map

$$
i: \Pi_{P, Q}\{\mathcal{P}(P, Q) \times F P, G Q\} \rightarrow \Pi_{P}\{F P, G Q\}
$$

arising from the idnetity $1 \rightarrow \mathcal{P}(P, P)$, and there are maps

$$
r, s, t: \Pi_{P, Q}\{\mathcal{P}(P, Q) \times F P, G Q\} \rightarrow \Pi_{P, Q, R}\{\mathcal{P}(Q, R) \times \mathcal{P}(P, Q) \times F P, G R\}
$$


arising respectively from $\mathcal{P}(Q, R) \rightarrow \mathcal{K}(G Q, G R)$, from the composition $\mathcal{P}(Q, R) \times$ $\mathcal{P}(P, Q) \rightarrow \mathcal{P}(P, R)$, and from $\mathcal{P}(Q, R) \rightarrow[F Q, F R]$. These maps satisfy

$$
i v=i w=1, \quad r v=s v, \quad r w=t v, \quad s w=t w
$$

so that what we have is a truncated co-simplicial object. Let $p: C \rightarrow \Pi_{P}\{F P, G P\}$, with $\lambda: v p \rightarrow w p$, be the inserter of $v$ and $w$. Let $h: D \rightarrow C$ be the equifier of the 2-cells

$$
r v p \underset{r \lambda}{\longrightarrow} r w p=t v p \underset{t \lambda}{\longrightarrow} t w p
$$

and

$$
r v p=s v p \underset{s \lambda}{\longrightarrow} s w p=t w p
$$

and let $k: E \rightarrow D$ be the equifier of the 2-cell

$$
p h=i v p h \underset{i \lambda h}{\longrightarrow} i w p h=p h
$$

and the identity. Then $E=\{F, G\}_{\ell}$, the unit being the lax natural $F \rightarrow \mathcal{K}(E, G-)$ whose data correspond to the components of $p h k$ and of $\lambda h k$. For (b), the construction is the same, except that $p$ is now the iso-inserter, and that the 2-cells to be equified are now invertible; this time we have $E=\{F, G\}_{p}$.

Combining this result with Propositions 4.2 and 4.4 gives:

Proposition 5.2. A 2-category that admits products, inserters, and equifiers, admits all lax limits and all pseudo-limits.

\section{INDEXED BILIMITS}

Since these have been discussed in detail in Section 1 of Street [15] - the error in his (1.24) and (1.25) being rectified in his [16] - we can be brief. We need the general notion, and such specialisations from bicategories to 2-categories as we shall use in [5] to prove the existence of bicolimits in 2-categories of algebras for a 2-monad.

From bicategories $\mathcal{P}$ and $\mathcal{K}$ we can form the bicategory $\operatorname{Hom}[\mathcal{P}, \mathcal{K}]$ whose objects are homomorphisms $\mathcal{P} \rightarrow \mathcal{K}$ of bicategories, whose arrows are strong transformations, and whose 2-cells are modifications. For the definitions of these terms, see Bénabou [2] and Street [15]; the strong transformations, and the modifications between them, are just the evident extensions to the bicategorical context of the pseudo-natural transformations of Section 5 above and the modifications between these. Accordingly, when $\mathcal{P}$ and $\mathcal{K}$ are in fact 2-categories, $\operatorname{Psd}[\mathcal{P}, \mathcal{K}]$ is a full sub-2-category of $\operatorname{Hom}[\mathcal{P}, \mathcal{K}]$; note that, for any bicategory $\mathcal{P}$, the bicategory $\operatorname{Hom}[\mathcal{P}, \mathcal{K}]$ is a 2-category if $\mathcal{K}$ is a 2-category. 
Given homomorphisms $F: \mathcal{P} \rightarrow$ Cat and $G: \mathcal{P} \rightarrow \mathcal{K}$ of bicategories, with $\mathcal{P}$ small, we have a homomorphism $\mathcal{K}^{o p} \rightarrow$ Cat sending $A$ to the right side of (6.1) below; when this homomorphism admits a birepresentation

$$
\mathcal{K}\left(A,\{F, G\}_{b}\right) \simeq \operatorname{Hom}[\mathcal{P}, \mathbf{C a t}](F, \mathcal{K}(A, G-))
$$

with unit say

$$
\zeta: F \rightarrow \mathcal{K}\left(\{F, G\}_{b}, G-\right)
$$

we call the object $\{F, G\}_{b}$ of $\mathcal{K}$, or more properly the pair $\left(\{F, G\}_{b}, \zeta\right)$, the $F$-indexed bilimit of $G$.

Recall from [15] that $\left(\{F, G\}_{b}, \zeta\right)$ is said to be a birepresentation of the homomorphism above when the functor from the left to the right side of $(6.1)$, sending $f$ to $\mathcal{K}(f, G-) \zeta$ and sending $\alpha: f \rightarrow g$ to $\mathcal{K}(\alpha, G-) \zeta$, is an equivalence of categories. (We use $\simeq$ for equivalences and reserve $\cong$ for isomorphisms.)

Note that we call a functor $T: \mathcal{A} \rightarrow \mathcal{B}$ an equivalence when there are a functor $S: \mathcal{B} \rightarrow \mathcal{A}$ and natural isomorphisms $\eta: 1 \cong T S$ and $\rho: S T \cong 1$; in which case, replacing $\rho$ by the isomorphism $\epsilon=\rho\left(S \eta^{-1} T\right)\left(\rho^{-1} S T\right)$, we in fact have an adjunction $\eta, \epsilon: S \rightarrow T: \mathcal{A} \rightarrow \mathcal{B}$. Such an equivalence $T$ is fully faithful and essentially surjective on objects; and the converse is true if our category Set of sets satisfies the axiom of choice. However, we deliberately refrain from imposing this axiom on Set, in order that our results may continue to hold in wider contexts. All the equivalences we establish in [5] are honest ones with explicit equivalence-inverses.

Because (6.1) (unlike (2.1), (5.3), and (5.4)) is only required to be an equivalence, the bilimit $\{F, G\}_{b}$ (unlike $\{F, G\},\{F, G\}_{\ell}$ and $\{F, G\}_{p}$ ) is determined only to within equivalence, and not to within isomorphism. Bicolimits in $\mathcal{K}$ are just bilimits in $\mathcal{K}^{o p}$, the bicolimit of $G: \mathcal{P} \rightarrow \mathcal{K}$ indexed by $F: \mathcal{P}^{o p} \rightarrow$ Cat being denoted by $F *_{b} G$. In the conical case $F=\Delta 1$, we can write bilim $G$ and bicolim $G$.

When $\mathcal{P}$ and $\mathcal{K}$ are 2-categories and $F$ and $G$ are 2-functors, we may by the fifth-last paragraph write $(6.1)$ as

$$
\mathcal{K}\left(A,\{F, G\}_{b}\right) \simeq \operatorname{Psd}[\mathcal{P}, \mathbf{C a t}](F, \mathcal{K}(A, G-))
$$

It follows from (5.7) that, if the pseudo-limit $\{F, G\}_{p}$ exists, it is a fortiori the bilimit $\{F, G\}_{b}$ - as is any object equivalent to it. Even in this case of 2 -functors, however, the bilimit may well exist when the pseudo-limit does not; a pseudo-initial-object in a 2-category is the same thing as an initial object, and it is easily seen that the 2-category Lex mentioned in Section 4 above has no initial object, although it has the category 1 as a bi-initial object. 
A bicategory $\mathcal{K}$ may be called bicategorically complete if it admits all bilimits $\{F, G\}_{b}$; the term "bicomplete" would be preferable, had it not been used elsewhere to mean "complete and cocomplete". Street shows in [16] that, although the construction in (1.24) of his [15] is wrong, the conclusion is after all correct: $\mathcal{K}$ admits all bilimits precisely when it admits biproducts, bi-equalizers, and bi-cotensor-products. For these three particular bilimits, $\mathcal{P}$ is merely a category, and the indexing type $F: \mathcal{P} \rightarrow$ Cat and the homomorphism $G: \mathcal{P} \rightarrow \mathcal{K}$ are honest functors; so that, when $\mathcal{K}$ is in fact a 2-category, these bilimits fall within the ambit of (6.3). We conclude that:

Proposition 6.1. A 2-category $\mathcal{K}$ admits all bilimits if and only if, for all 2functors $F: \mathcal{P} \rightarrow$ Cat and $G: \mathcal{P} \rightarrow \mathcal{K}$ with $\mathcal{P}$ small, we have a birepresentation (6.3); it suffices in fact to suppose $\mathcal{P}$ merely a category.

A homomorphism $N: \mathcal{L} \rightarrow \mathcal{K}$ of bicategories is said to be a left biadjoint of the homomorphism $M: \mathcal{K} \rightarrow \mathcal{L}$ if there is an equivalence $\mathcal{K}(N ?,-) \simeq \mathcal{L}($ ?, $M-)$ in $\operatorname{Hom}\left[\mathcal{L}^{\text {op }}, \operatorname{Hom}[\mathcal{K}, \mathbf{C a t}]\right]$. Such a left biadjoint $N$ of $M$ is unique to within equivalence if it exists; and it exists exactly when each $\mathcal{L}(B, M-): \mathcal{K} \rightarrow$ Cat admits a birepresentation. $\{$ This precise formulation is not given explicitly in (1.30) of [15], but it can be checked without too much trouble. The details will be given in [11]. $\}$ Even when $M$ is a 2-functor between 2-categories, it may adnit a left biadjoint $N$ although admitting no left adjoint; then, of course, it is not in general possible so to choose $N$ that it is a 2-functor.

This language allows an alternative formulation of Proposition 6.1; we write it in the colimit form in which we shall apply it in [5]. Recall the 2 -functor $\widetilde{G}: \mathcal{K} \rightarrow\left[\mathcal{P}^{\circ p}\right.$, Cat $]$ of (2.8) induced by $G: \mathcal{P} \rightarrow \mathcal{K}$, and write $J:\left[\mathcal{P}^{o p}\right.$, Cat $] \rightarrow \operatorname{Psd}\left[\mathcal{P}^{o p}\right.$, Cat $]$ for the inclusion 2-functor.

Proposition 6.2. A 2-category $\mathcal{K}$ admits all bicolimits if and only if, for every 2-functor $G: \mathcal{P} \rightarrow \mathcal{K}$ with $\mathcal{P}$ small, the 2-functor $J \tilde{G}: \mathcal{K} \rightarrow \operatorname{Psd}\left[\mathcal{P}^{\circ p}\right.$, Cat] has a left biadjoint; it suffices in fact to suppose $\mathcal{P}$ merely a category.

\section{References}

[1] C. Auderset, 'Adjonctions et monades au niveau des 2-catégories', Cahiers de Topologie et Géom. Diff. 15 (1974), 3-20.

[2] J. Bénabou, 'Introduction to bicategories', in Lecture Notes in Math. 47, pp. 1-77 (Springer-Verlag, Berlin, Heidelberg, New York, 1967).

[3] G.J. Bird, Limits of Locally-presentable Categories (Ph.D thesis, Univ. of Sydney, 1984).

[4] G.J. Bird, G.M. Kelly, A.J. Power and R. Street, 'Flexible limits for 2-categories', J. Pure Appl. Algebra (to appear).

[5] R. Blackwell, G.M. Kelly and J. Power, 'Two-dimensional monad theory', J. Pure Appl Algebra (to appear). 
[6] F. Borceux and G.M. Kelly, 'A notion of limit for enriched categories', Bull. Austral. Math. Soc. 12 (1975), 49-72.

[7] J.W. Gray, 'Formal Category Theory : Adjointness for 2-Categories': Lecture Notes in Math. 391 (Springer-Verlag, Berlin, Heidelberg, New York).

[8] G.M. Kelly, 'On clubs and doctrines', in Lecture Notes in Math. 420 (Springer-Verlag, Berlin, Heidelberg, New York, 1974). pp. 181-256.

[9] G.M. Kelly, Basic Concepts of Enriched Category Theory (London Math Soc. Lecture Notes Series 64, Cambridge Univ. Press, 1982).

[10] G.M. Kelly, 'Structures defined by fnite limits in the enriched context I', Cahiers de Topologie et Géom. Diff 23 (1982), 3-42.

[11] G.M. Kelly, 'Equivalences in 2-categories, birepresentations, and biadjoints', (in preparation).

[12] G.M. Kelly and R. Street, 'Review of the elements of 2-categories', in Lecture Notes in Math. 420 (Springer-Verlag, Berlin, Heidelberg, New York, 1974). pp. 75-103.

[13] R. Street, 'Elementary Cosmoi I', in Lecture Notes in Math 420 (Springer-Verlag, Berlin, Heidelberg, New York, 1974). pp. 134-180.

[14] R. Street, 'Limits indexed by category-valued 2-functor', J. Pure Appl. Algebra 8 (1976), $149-181$.

[15] R. Street, 'Fibrations in bicategories', Cahiers de Topologie et Géom. Diff. 21 (1980), 111-160.

[16] R. Street, "Corrigendum to "Fibrations in bicategories"', Cahiers de Topologie et Géom. Diff. Catégoriques 28 (1987), 53-56.

Pure Mathematics Department.

University of Sydney

N.S.W. 2006

Australis 